\title{
HIGHLIGHTS
}

THERAPY

\section{Rituximab has similar short-term safety and efficacy to cyclophosphamide in ANCA-associated vasculitis}

Cyclophosphamide and glucocorticoids are the mainstay of induction therapy for antineutrophil cytoplasmic antibody (ANCA)-associated vasculitis; however, this treatment is associated with high mortality and serious adverse events. New results indicate that rituximab, an anti-CD20 monoclonal antibody, has similar safety and efficacy to cyclophosphamide in this setting, at least in the short term.

In a multicenter, double-blind, doubledummy, noninferiority trial, Stone et al. randomly assigned patients with severe, newly diagnosed or relapsing disease to receive standard glucocorticoid therapy plus either rituximab $(n=99)$ or cyclophosphamide $(n=98)$. Adverse event rates did not differ between these groups, and similar proportions of patients achieved steroid-free remission at 6 months. However, the rituximabbased regimen more effectively induced remission in patients with relapsing disease; 34 of 51 such patients treated with rituximab achieved steroid-free remission, compared with 21 of 50 those treated with cyclophosphamide.

Jones and colleagues report similar and complementary findings in an open-label, parallel-design, randomized trial. Patients with newly diagnosed, severe disease received either rituximab with two doses of cyclophosphamide and glucocorticoids $(n=33)$ or cyclophosphamide and glucocorticoids $(n=11)$. Rates of severe adverse events and sustained remission were similar at 12 months in both groups.

Rituximab-based therapy did not improve either the safety or efficacy of induction treatment for ANCA-associated vasculitis in the two studies. Nonetheless, the researchers remain optimistic about the potential long-term benefits, most likely to be related to a decreased need for maintenance immunosuppression and reduced cumulative exposure to cyclophosphamide. Stone et al. and Jones et al. thus plan to monitor their patients for $\geq 18$ months and $\geq 24$ months,

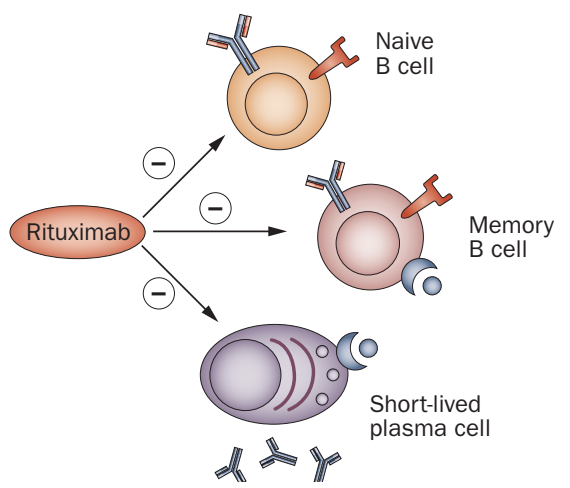

respectively. Rituximab could be a valuable option for patients at the greatest risk of cyclophosphamide-related adverse effects and might even become the first choice for the treatment of relapsing disease.

Shreeya Nanda

Original articles Jones, R. B. et al. Rituximab versus cyclophosphamide in ANCA-associated renal vasculitis. N. Engl. J. Med. 363, 211-220 (2010) | Stone, J. H. et al. Rituximab versus cyclophosphamide for ANCA-associated vasculitis. N. Engl. J. Med. 363, 221-232 (2010) 